\title{
Biomarkers: Where to Go from Here
}

\author{
Angelo Paradiso \\ National Cancer Research Center, Istituto Tumori 'Giovanni Paolo II', Bari, Italy
}

In this issue of BREAST CARE, four articles focus on various bio-pathological issues of potentially great relevance from the prognostic-predictive point of view:

In a large series of Chinese women with breast cancer, Zhang and colleagues [1] analyzed the pathological characteristics of triple-negative breast cancer (TNBC) in order to provide a more reliable basis for their clinical treatment. The authors report a prevalence of TNBC in Chinese ethnicity similar to that already reported by others [2], however, at multivariate analysis a worse prognosis for this subset of breast cancer patients was not confirmed in either disease free or overall survival. The diversity of primary local and systemic therapy the patients received and the retrospective characteristics of almost all published studies are the main reasons why it is difficult to interpret the controversial results available in the literature concerning the prognosis of TNBC women. Ad hoc prospective studies urgently need to be activated to answer the question of clinical aggressiveness of TNBC and, above all, to individualize optimal local and systemic treatment for this particular subset of patients.

The interest of scientists in the 70-gene prognostic signature Mammaprint ${ }^{\circledR}$ applied to breast cancer patients increases. In this issue, Hartmann and colleagues [3] compare the prognostic relevance of Mammaprint with the clincal risk assessment according to Adjuvant! Online (AoL) in a prospective cohort of patients older than 60 years; the authors report an important discordance rate $(48 \%)$ between the prognostic categorization of the profile and AoL while the recent result [4] on feasibility of the EORTC 10041/BIG 03-04 MINDACT trial reported a disagreement between clinical criteria (AoL) and Mammaprint of only 27\%. Whether the higher discordance value reported by Hartmann et al. is due to the limited number of patients enrolled in their study, to technical factors, or really to the different performances of the two prognostic tools when applied to elderly patients still needs to be definitely ascertained.

Circulating tumor cells (CTCs) have recently captured the spotlight because of their exciting potential role as prognostic markers for the clinical management of cancer patients [5]. However, translation of CTC technologies into clinical practice still requires progress in assay development and in definitively establishing its clinical utility. In fact, even though some prospective studies already support the idea that these cells are highly relevant for the prediction of metastatic spread, isolation of these cells has proved to be delicate because of the low rate of CTC events. In this issue, de Albuquerque and colleagues [6] suggest the use of a panel of protein markers for CTC isolation and identification, which showed good performance in their small prospective study. By immunomagnetic tumor cell enrichment and an RT-PCR approach for 6 markers, the authors obtained a 56\% sensitivity and 100\% specificity in metastatic patients compared to healthy controls. Interestingly, Her-2/neu was included in the panel of markers for CTC characterization, showing positive results in an appreciable percentage of cases. The authors concluded that the multimarker characterization of CTC cells is feasible and that this approach could also contribute towards tailoring a more individualized therapy. In their opinion, the high consistency shown for their multimarker analysis calls for larger confirmatory studies.

The effect of the CYP2D6 genotypic variation on tamoxifen (TAM) metabolism is one of the best characterized and most clinically important examples of pharmacogenomics in cancer. However, a recent systematic review [7] on this argument concluded that data were limited and conflicting and that therefore it was still not possible to recommend pharmacogenetic testing in breast cancer patient populations. The authors recommend further research to focus also on which genotypes reflect patient response and on the possibility of using more reliable surrogate markers for CYP2D6 activity. In this issue of BREAST CARE, Trojan and colleagues [8] report the results of their study confirming the possibility of using urinary dextromethorphan phenotyping as a standardized tool to better assess CYP2D6 metabolic capacity. The authors suggest this method is efficient and reproducible and, as a consequence, could be a better and easier tool to assess

\section{KARGER}

Fax +497614520714

Information@Karger.de

www.karger.com (c) 2012 S. Karger GmbH, Freiburg

1661-3791/12/0071-0005\$38.00/0

Accessible online at:

www.karger.com/brc
Angelo Paradiso, MD

National Cancer Research Cente

Istituto Tumori 'Giovanni Paolo II'

Viale O. Flacco, 65, 70125 Bari, Italy

Tel. +39080 5555-900, Fax -388

a.paradiso@oncologico.bari.it 
CYPD26 metabolic capacity than endoxifen formation or extensive CYP2D6 genotyping. These authors also call for prospective studies to confirm their hypothesis.

What do all these studies have in common? They are all good examples of translational research in the specific field of breast cancer, but they are also calling strongly for prompt clinical validation and/or application, of a new taxonomy, an innovative biomolecular profiling, a pharmacogenomic information, etc.

The scientific literature is full of promising biomarkers 'lost in translation' towards clinical application. Therefore, the priority is to make the validation process for new biomarkers more efficient. From this point of view, a closer collaboration and cross-talk between the laboratory and clinics is mandatory; common go/no go and prioritization criteria should be established for the selection of biomarkers entering the validation process; common tools, such as tissue biobanks, data management, and reference materials should be available. This is the vision that is in development in Europe and the US.

Recently, a German Consortium for Translational Cancer Research has been established which offers ideal conditions to pursue this line. The partnership of the German Cancer Research Center with 7 university sites will surely provide know-how and tools to help current research results translate even more swiftly into clinical practice.

In the US, perhaps the best example of a Network for biomarker development is represented by the Early Detection Research Network (EDRN) of NCI-NIH (http://edrn.nci.nih. gov/). This network was created in 2000 to muster a strong, investigator-driven force to conduct translational research to identify tests for early cancer and cancer risk ready for largescale clinical testing. Now dozens of biomarker developmental laboratories, biomarker reference laboratories, clinical epidemiology and validation centers work together to provide biospecimens, reference materials, and bio-informatics knowhow to facilitate the development of the complicated life of a biomarker.

\section{Disclosure Statement}

The author has no conflicts of interests to declare.

\section{References}

1 Zhang L, Hao C, Dong G, Tong Z: Analysis of clincial features and outcome of 356 triple-negative breast cancer in China. Breast Care 2012;7: DOI: $10.1159 / 000336539$

- 2 Su Y, Zheng Y, Zheng W, Gu K, Chen Z, Li G, Cai Q, Lu W et al.: Distinct distribution and prognostic significance of molecular subtypes of breast cancer in Chinese women: a population-based cohort study. BMC Cancer 2011;11:292.

3 Hartmann S, Gerber B, Elling D, Heintze K, Reimer T: The 70-gene signature as prognostic factor for elederly women with hormone receptor-positive, HER2negative breast cancer. Breast Care 2012;7: DOI: 10.1159/000336552.

4 Rutgers E, Piccart-Gebhart MJ, Bogaerts J, Delaloge S, Veer LV, Rubio IT, Viale G, Thompson AM, Passalacqua R, Nitz U, Vindevoghel A, Pierga JY, Ravdin PM, Werutsky G, Cardoso F. The EORTC 10041/BIG 03-04 MINDACT trial is feasible: results of the pilot phase. Eur J Cancer 2011;47:2742-2749.

5 Sleijfer S, Foekens JA: Medical oncology: clinical value of circulating tumor cells in breast cancer. Nat Rev Clin Oncol 2011;8:696-698.

6 de Albuquerque A, Kaul S, Breier G, Krabisch P, Fersis N: Multimarker analysis of circulating tumour cells in perpheral blood of metastatic breast cancer patients - a step forward in personalized medicine. Breast Care 2012;7: DOI: $10.1159 / 000336548$

7 Fleeman N, Martin Saborido C, Payne K, Boland A, Dickson R, Dundar Y, Fernández Santander A, Howell S, Newman W, Oyee J, Walley T: The clinical effectiveness and cost-effectiveness of genotyping for CYP2D6 for the management of women with breast cancer treated with tamoxifen: a systematic review. Health Technol Assess 2011;15:1-102.

8 Trojan A, Vergopoulos A, Breitenstein U, Seifert B, Rageth C, Joechle W: The discriminatory value of CYP2D6 genotyping in predicting the dextromethorphan phenotype in women with breast cancer. Breast Care 2012;7: DOI: $\underline{10.1159 / 000336551}$ 